\title{
Results from a multimedia testing and counseling program in an urban emergency department
}

\author{
Yvette Calderon ${ }^{1,2}$, Ethan Cowan ${ }^{1,2}$, Jade Fettig ${ }^{1 *}$, Karachi Egbuta ${ }^{1}$, Jason Leider ${ }^{1,2}$ \\ From $16^{\text {th }}$ International Symposium on HIV and Emerging Infectious Diseases \\ Marseille, France. 24-26 March 2010
}

\section{Background}

This study evaluates a novel approach to counseling and testing in a high-volume inner-city ED which utilizes an HIV counselor and a multimedia tool for conveying video HIV information and electronically collecting risk factor data. We evaluated this program to assess the demographic and risk factor characteristics of all patients tested, patient-reported satisfaction with the program and outcomes for positive patients.

\section{Methods}

This prospective cross-sectional evaluation was conducted for 2 years. A convenience sample of medically stable patients presenting to an inner-city municipal hospital ED were recruited by 3 to 8 full-time equivalent HIV counselors. Previously developed and validated videos for HIV pre- and post-test counseling were used. Demographic characteristics, risk factors, and satisfaction information were collected using patient self-reporting on the touch screen computer. Data downloaded automatically into a secure database. Chart reviews were conducted by the HIV-positive patients' medical provider to assess outcomes. Data were analyzed using SPSS software.

\section{Results}

During the federal grant period, 28,995 patients were tested for HIV. Demographic characteristics of the participants were: $41.9 \%$ male, mean age $36.0 \pm 14.2$ years, 54.7\% Hispanic, and 32.2\% African-American. Risk factors were: $6.4 \%$ MSM, $31.0 \%$ had multiple sex partners in the past 3 months, $49.8 \%$ reported condom use as "never," $1.5 \%$ used injection drugs. Patient satisfaction was high: $88.8 \%$ reported learning a moderate-to-large amount of new information about HIV and $78.6 \%$ preferred the format which included both videos and an HIV counselor. There were 101 newly diagnosed or confirmed HIV positive patients and $86 \%$ were linked to outpatient HIV care; mean days to first medical visit was 7. Positive patient outcomes were as follows: $85 \%$ of eligible patients began HAART, median days to HAART treatment was $35,62 \%$ of patients on HAART had viral load less than 400 copies $/ \mathrm{mL}$.

\section{Discussion}

A rapid HIV program using a multimedia tool and a counselor in a busy inner-city hospital ED can effectively test a large number of patients, provide consistent prevention messages to patients who report multiple HIV risk factors and link a large percentage HIV-positive patients to existing health care systems.

\section{Author details}

'Jacobi Medical Center, New York, USA. ${ }^{2}$ Albert Einstein College of Medicine, New York, USA.

Published: 11 May 2010

doi:10.1186/1742-4690-7-S1-P97

Cite this article as: Calderon et al:: Results from a multimedia testing

and counseling program in an urban emergency department.

Retrovirology 2010 7(Suppl 1):P97.

* Correspondence: jade.fettig@nbhn.net

'Jacobi Medical Center, New York, USA 